\title{
Analytical study of the reflection and transmission coefficient of the submarine interface
}

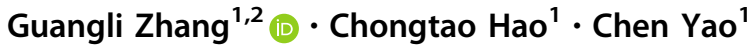

Received: 22 August 2017 / Accepted: 27 April 2018/Published online: 10 May 2018

(C) The Author(s) 2018

\begin{abstract}
The analytical study of the reflection and transmission coefficient of the seafloor interface is essential for the characterization of the ocean bottom in marine seismic exploration. Based on the boundary conditions of the seafloor interface, the analytical expression of the reflection and transmission coefficient at the submarine interface is derived in this study by using the steady-state wave solution of the elastic wave in a homogeneous, isotropic medium. With this analytical expression, the characteristics of the reflection and transmission coefficient at the submarine interface are analysed and discussed using critical angles. The results show that the change in the reflection and transmission coefficient with the incidence angle presents a "segmented" characteristic, in which the critical angle is the dividing point. The amplitude value and phase angle of the coefficient at the submarine interface change dramatically at the critical angle, which is related to the P- and S-wave velocities in the seabed layer. Compared with the stiff seabed, the soft seabed has a larger P-wave critical angle and an absence of the converted S-wave critical angle, owing to the low P-and S-wave velocities in the solid seabed layer. By analysing and discussing the special changes that occur in the coefficient values at the critical angle, the reflection and transmission characteristics of the different incident angles are obtained. Synthetic models of both stiff and soft seafloors are provided in this study to verify the analytical results. Finally, we compared our synthetic results with real data from the Gulf of Mexico, which enabled the validation of our conclusions.
\end{abstract}

Keywords Submarine interface - Reflection and transmission coefficient - Stiff and soft seabed layer - Critical angle Analytical study

\section{Introduction}

The analytical study of the reflection and transmission coefficient of the submarine interface is essential for characterizing the ocean bottom. Zoeppritz (1919) first expressed the reflection and transmission coefficient as a function of the incidence angle and elastic medium parameters. To address this nonlinear problem, linear approximations of Zoeppritz equations were derived for analytical study under different assumptions, such as a

Guangli Zhang

glzhang@mail.iggcas.ac.cn

1 State Key Laboratory of Earthquake Dynamics, Institute of Geology, Earthquake Administration China, Beijing, China

2 Key Laboratory of Earth and Planetary Physics, Institute of Geology and Geophysics, Chinese Academy of Sciences, Beijing 10029, China weak property contrast and limited incident angles (e.g. Bortfeld 1961; Aki and Richards 1980; Shuey 1985; Xu and Bancroft 1997; Zhu and McMechan 2012; Zhao and Shen 2013). Regarding the submarine interface, the seismic wave propagates as an acoustic wave in the sea water and changes into an elastic wave in the seabed layer. The properties across the submarine interface change dramatically in the wide-angle domain because the two highcontrast media are in contact with each other, which makes the linear approximations not valid for submarine interfaces (Bull et al. 1998; Zong et al. 2013; Yin et al. 2013; Liu et al. 2015; Aleardi and Tognarelli 2016). Therefore, the analytical study of the reflection and transmission coefficient of the submarine interface must be based directly on the nonlinear problem discussion.

Previously, to solve this nonlinear problem, a considerable amount of research was conducted. Kennett (1979) first recognized and discussed the specificity of the 
reflection and transmission coefficient in the seabed interface. Crampin (1981) modified the propagation equation form by associating the displacement-stress vectors at the top interface with the bottom interface of the aquifer. Mallick and Frazer (1991) derived formulae for the reflection and transmission coefficients at the interfaces between liquids and azimuthally anisotropic solids. Carcione (2001) obtained the matrix equations of the submarine interface by modifying the Zoeppritz equation boundary conditions. The equations obtained from these studies allowed calculating reflection and transmission coefficients for submarine interfaces. But, the exact analytical expressions for these coefficients for these coefficients were not provided in these works. And, we believe that providing these expressions can improve our understanding of the seafloor reflection and transmission.

Liu and Liu (2015) derived a nonlinear analytical expression for the $\mathrm{P}$-wave reflection coefficient at the seafloor interface for the amplitude variation with offset (AVO) inversion, but the expression form provided is not available at critical angles because there is a transmission angle cosine on the denominator, and thus, only the cases in which the incident angles were less than the critical angle were discussed. However, critical angles at the submarine interface dominate the variation in the reflection and transmission coefficient of the seafloor interface (Riedel and Theilen 2001), and the properties of the P-wave at critical angles are also essential for both the interpretation and inversion problems of reflection (Ruud 2006; Kanaun et al. 2012; Zhu and Mcmechan 2012). In particular, with the gradual marine seismic exploration transformation from shallow water to a deep marine environment, there is a growing number of critical angles in the real data for large offset situations (Downton and Ursenbach 2006; Skopintseva et al. 2011). Hence, an exact expression, which can be used to discuss critical angles in the submarine interface, is of urgent need for the analytical study of the P-wave reflection and transmission coefficient.

The variation in the reflection and transmission coefficient with the offset could be a direct indicator of the medium properties (Ostrander 1984). Therefore, the AVO, or amplitude variation with incidence angle (AVA) was conducted to predict the lithology and fluid properties (Castagna and Backus 1993; Riedel et al. 2003; Sun et al. 2008; Alemie and Sacchi 2011; Lu et al. 2015). The analytical study of the reflection and transmission coefficient of the submarine interface, which can provide a theoretical basis for AVO or AVA, has a high demand for analytical forms and analysability. In this paper, we deduced an analytical expression for both the reflection and transmission coefficients at the submarine interface in the form of the wave impedance, incidence angle and transmission angle. Compared with conventional approximations of the
Zoeppritz equations, the analytical expression we derived has an accurate value in the wide-angle domain of the submarine interface. Considering the slowness of the horizontal waves and steady-state wave solutions of the seismic waves on both sides of the seafloor interface, the expression form is applicable to the critical angle. With this expression, we can discuss the reflection and transmission properties at different critical angles. Therefore, we can obtain the overall variation features of the reflection and transmission coefficients with different submarine parameters through the analytical study of these critical angles to resolve this nonlinear problem. Given that it is not possible to trap the energy at the critical angle, energy normalization is also necessary for the analytical expression of seafloor coefficients (Kennett 1979; Aki and Richards 1980). Finally, two methods were used to validate our analytical expression and results: one is the establishment of typical stiff and soft seafloor models, and the other is the comparison of synthetic results with real data from the Gulf of Mexico.

\section{Methodology}

\section{Steady-state wave solution}

As shown in Fig. 1, the submarine interface is approximately considered to be a liquid-solid interface on both sides of the homogeneous semi-infinite space. The upper space is the liquid medium, and the lower space denotes a solid medium. Parameters $\rho_{1}$ and $\alpha_{1}$ represent the density and velocity of the seawater; and $\rho_{2}, \alpha_{2}$ and $\beta_{2}$ denote the density, P-wave velocity and $\mathrm{S}$-wave velocity in a solid medium. Considering that there is no S-wave in the upper layer, we assume that three possible incident waves are

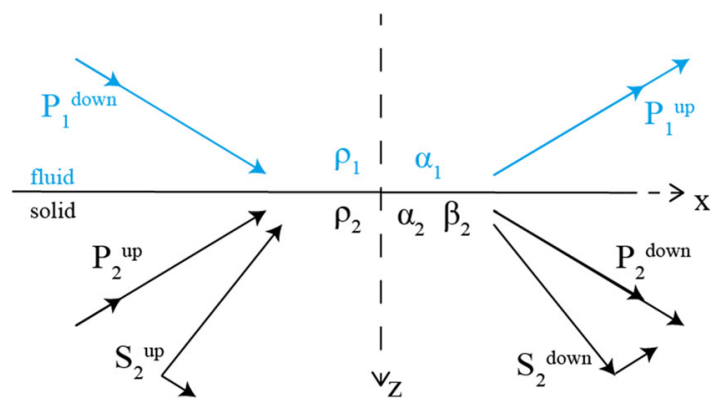

Fig. 1 Incidence, reflection and transmission waves at the fluid-solid interface. $P_{1}^{\text {down }}, P_{2}^{\text {up }}$ and $S_{2}^{\text {up }}$ denote the displacement amplitude of different incident waves. $P_{1}^{\text {up }}, P_{2}^{\text {down }}$ and $S_{2}^{\text {down }}$ represent the displacement amplitudes of the scattered waves. Subscripts 1 and 2 represent the liquid medium and solid medium, respectively. Short, solid arrows show the direction of particle motion; long, solid arrows express the direction of propagation; and dashed arrows indicate the directions of the $x$ - and $z$-axes 
present together and have the same horizontal slowness at this interface. In Fig. $1, P_{1}^{\text {down }}, P_{2}^{\text {up }}$ and $S_{2}^{\text {up }}$ represent the displacement amplitudes of the incident waves; $P_{1}^{\text {up }}, P_{2}^{\text {down }}$ and $S_{2}^{\text {down }}$ represent the displacement amplitudes of the scattered waves, where subscripts 1 and 2 represent the seawater and solid layer, respectively. The direction of horizontal slowness increase is defined as the positive direction of the $x$-axis, and the vertical downward direction is the positive $z$-axis.

The wave equation for an elastic wave in a homogeneous medium can be expressed as follows:

$\alpha^{2} \nabla^{2} \phi=\frac{\partial^{2} \phi}{\partial^{2} t}$

In Eq. (1), $t$ is time, $\alpha$ is the velocity and $\phi$ is the potential for plane waves. The steady-state plane wave solution of Eq. (1) assumes the form of $\left.A \mathrm{e}^{i(\vec{k}} \vec{x}-\omega t\right)$, where $A$ and $\omega$ are the amplitude and frequency, respectively, and $\vec{k}=\left(k_{x}, k_{y}, k_{z}\right)$ is the wave number vector, which satisfies $|k|=\frac{\omega}{\alpha}$.

With the steady-state wave solutions, the vector displacements of all wave types in Fig. 1 are expressed in Table 1. In Table 1, $p$ represents the ray parameter at the submarine interface; $i_{1}$ represents the incident angle of the downward incidence P-wave in the sea water; $i_{2}$ represents the transmission angle of the transmitted P-wave; and $j_{2}$ represents the transmission angle of the converted S-wave in the solid seabed layer. With Snell's law, the relationship between these angles and the ray parameter is as follows:

$\frac{\sin i_{1}}{\alpha_{1}}=\frac{\sin i_{2}}{\alpha_{2}}=\frac{\sin j_{2}}{\beta_{2}}=p$

\section{Boundary conditions}

Although we have obtained the wave vector displacements at the submarine interface, we need boundary conditions to associate the displacements in the equations. The liquidsolid interface has a displacement discontinuity in the tangential component due to the low seawater viscosity (Aki and Richards 1980; Sidler and Carcione 2007). In addition, the shear stress vanished at the liquid-solid interface, and thus, there is only the continuity of the normal displacement and normal stress at the submarine interface. Therefore, the boundary conditions of the submarine interface can be expressed as follows:

$$
\left\{\begin{array}{l}
\frac{\partial \phi_{1}}{\partial z}=\frac{\partial \phi_{2}}{\partial z} \\
\lambda_{1} \nabla^{2} \phi_{1}+2 \mu_{1} \frac{\partial^{2} \phi_{1}}{\partial z^{2}}=\lambda_{2} \nabla^{2} \phi_{2}+2 \mu_{2} \frac{\partial^{2} \phi_{2}}{\partial z^{2}} \\
2 \mu_{1} \frac{\partial^{2} \phi_{1}}{\partial z \partial x}=2 \mu_{2} \frac{\partial^{2} \phi_{2}}{\partial z \partial x}
\end{array}\right.
$$

In Eq. (3), $\phi_{1}$ and $\phi_{2}$ represent the potential wave functions of the two media, respectively; $\lambda_{1}, \mu_{1}, \lambda_{2}$ and $\mu_{2}$ represent the Lame constants on both sides of the seafloor interface; and subscripts 1 and 2 represent the liquid medium and solid medium, respectively. In the case of stress calculations, we replace the Lame constants $\lambda$ and $\mu$ as follows:

$\mu=\rho \beta^{2}, \lambda=\rho \alpha^{2}-2 \rho \beta^{2}$

With these three boundary conditions, three equations can be obtained by substituting the wave vector displacements in Table 1 into the boundary condition in Eq. (3). The three equations are as follows:

$\left\{\begin{array}{c}\cos i_{1}\left(P_{1}^{\text {down }}-P_{1}^{u p}\right)=\cos i_{2}\left(P_{2}^{\text {down }}-P_{2}^{u p}\right)-\sin j_{2}\left(S_{2}^{\text {down }}-S_{2}^{\text {up }}\right), \\ \rho_{1} \alpha_{1}\left(P_{2}^{\text {down }}+P_{2}^{u p}\right)=\rho_{2} \alpha_{2}\left(1-2 \beta_{2}^{2} p^{2}\right)\left(P_{2}^{\text {down }}+P_{2}^{u p}\right)-2 \rho_{2} \beta_{2}^{2} p \cos j_{2}\left(S_{2}^{\text {down }}+S_{2}^{u p}\right), \\ 0=2 \rho_{2} \beta_{2}^{2} p \cos i_{2}\left(P_{2}^{\text {down }}-P_{2}^{u p}\right)+\rho_{2} \beta_{2}\left(1-2 \beta_{2}^{2} p^{2}\right)\left(S_{2}^{\text {down }}-S_{2}^{\text {up }}\right) .\end{array}\right.$

\section{Solution of the coefficient matrix}

Rearranging Eq. (5), the incident waves are all on the right side and the scattered waves are all on the left, and thus, we obtain the following:

$\mathbf{M}\left(\begin{array}{c}P_{1}^{\text {up }} \\ P_{2}^{\text {down }} \\ S_{2}^{\text {down }}\end{array}\right)=\mathbf{N}\left(\begin{array}{c}P_{1}^{\text {down }} \\ P_{2}^{\text {up }} \\ S_{2}^{\text {up }}\end{array}\right)$

where $\mathbf{M}$ and $\mathbf{N}$ are the coefficient matrices of the submarine interface, respectively. The concrete expressions of the matrices are as follows:

$\mathbf{M}=\left(\begin{array}{ccc}\cos i_{1} & \cos i_{2} & -\beta_{2} p \\ 0 & 2 \rho_{2} \beta_{2}^{2} p \cos i_{2} & \rho_{2} \beta_{2}\left(1-2 \beta_{2}^{2} p^{2}\right) \\ -\rho_{1} \alpha_{1} & \rho_{2} \alpha_{2}\left(1-2 \beta_{2}^{2} p^{2}\right) & -2 \rho_{2} \beta_{2}^{2} p \cos j_{2}\end{array}\right)$

$\mathbf{N}=\left(\begin{array}{ccc}\cos i_{1} & \cos i_{2} & -\beta_{2} p \\ 0 & 2 \rho_{2} \beta_{2}^{2} p \cos i_{2} & \rho_{2} \beta_{2}\left(1-2 \beta_{2}^{2} p^{2}\right) \\ \rho_{1} \alpha_{1} & -\rho_{2} \alpha_{2}\left(1-2 \beta_{2}^{2} p^{2}\right) & 2 \rho_{2} \beta_{2}^{2} p \cos j_{2}\end{array}\right)$

With Eq. (7), we can obtain the inverse matrix $\mathbf{M}^{-1}$ of $\mathbf{M}$, and the expression of $\mathbf{M}^{-1}$ is as follows:

$\mathbf{M}^{-1}=\frac{1}{E}\left(\begin{array}{ccc}b & F+d \cos j_{2} & \rho_{2} \beta_{2} \cos i_{2} \\ c-a & -\rho_{1} \alpha_{1} \beta_{2} p-d \cos j_{2} & -\rho_{2} \beta_{2} \cos i_{1}+\frac{c \cos i_{1}}{\rho_{1} \alpha_{1}} \\ \rho_{1} \alpha_{1} d \frac{c \alpha_{2}}{\rho_{1} \beta_{2}}-\rho_{1} \beta_{1} \cos i_{2}-\rho_{2} \beta_{2} \cos i_{1} & d \cos i_{1}\end{array}\right)$

where $a, b, c, d, E$ and $F$ are intermediate variables defined as follows: 
Table 1 P-SV wave vector displacement at the seafloor interface

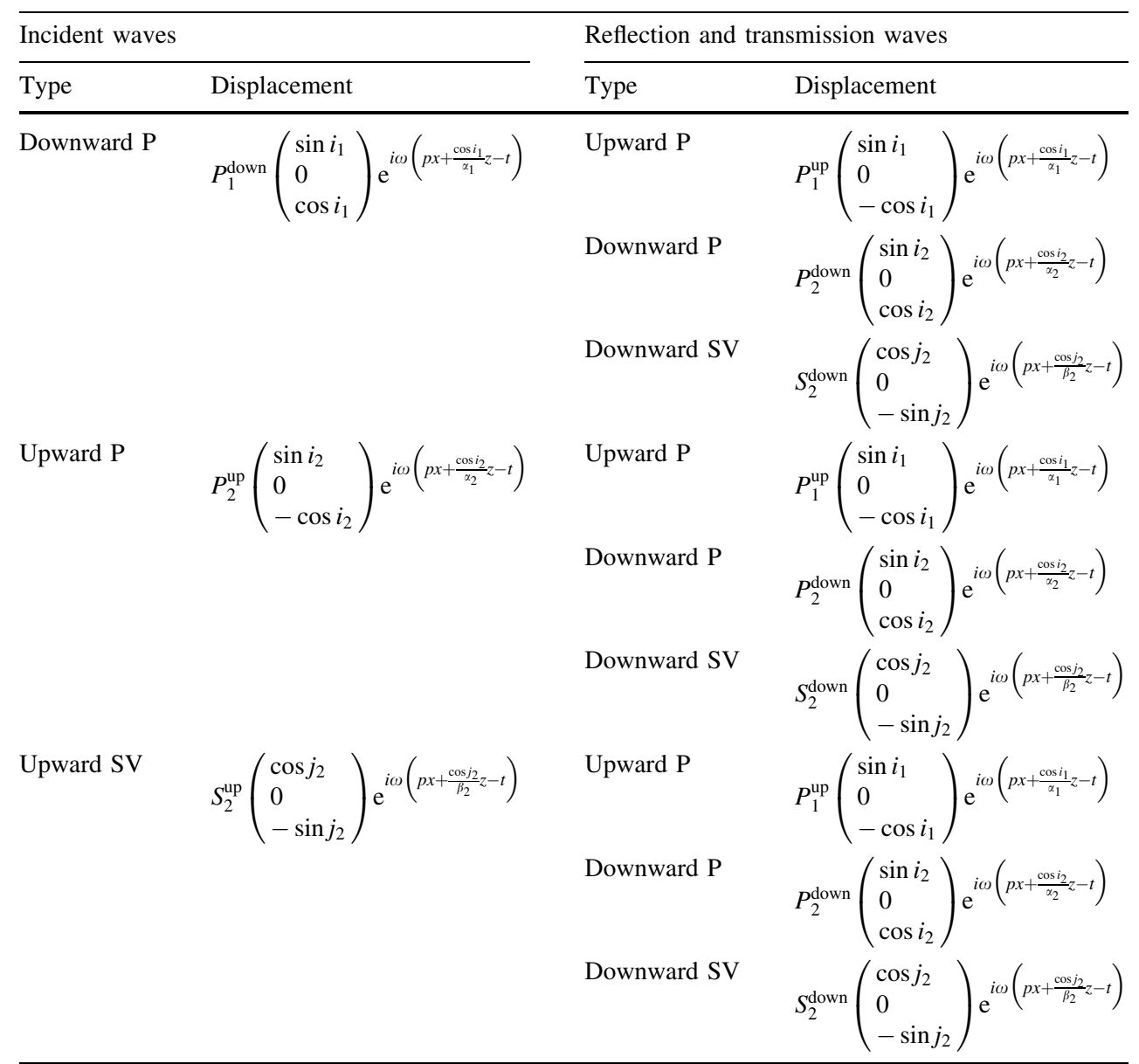

$a=\alpha_{1} \beta_{2} \rho_{1} \rho_{2}$,

$b=-\alpha_{2} \beta_{2} \rho_{2}^{2}+4 p^{2} \alpha_{2} \beta_{2}^{3} \rho_{2}^{2}-4 p^{2} \cos i_{2} \cos j_{2} \beta_{2}^{4} \rho_{2}^{2}-4 p^{4} \alpha_{2} \beta_{2}^{5} \rho_{2}^{2}$,

$c=2 p^{2} \alpha_{1} \beta_{2}^{3} \rho_{1} \rho_{2}$,

$d=2 p \cos i_{2} \beta_{2}^{2} \rho_{2}$,

$E=b \cos i_{1}-a \cos i_{2}$

$F=p \alpha_{2}\left(\frac{c}{\rho_{1} \alpha_{1}}-\rho_{2} \beta_{2}\right)$

According to Eqs. (6), (8) and (9), the scattering matrix of the reflection and transmission coefficient of the submarine interface can be obtained as follows:

$\mathbf{A}=\mathbf{M}^{-1} \mathbf{N}=\left(\begin{array}{lll}P^{d} P^{u} & P^{u} P^{u} & S^{u} P^{u} \\ P^{d} P^{d} & P^{u} P^{d} & S^{u} P^{d} \\ P^{d} S^{d} & P^{u} S^{d} & S^{u} S^{d}\end{array}\right)$

In Eq. (11), each column of the matrix $\mathbf{A}$ represents the three waves scattered away from the interface by a type of incident wave at the submarine interface. Among the waves, $P^{\mathrm{d}} P^{\mathrm{u}}, P^{\mathrm{d}} P^{\mathrm{d}}$ and $P^{\mathrm{d}} S^{\mathrm{d}}$ represent the reflected and transmitted waves generated by the downward incident $\mathrm{P}$ - wave in the sea water; $P^{\mathrm{u}} P^{\mathrm{d}}, P^{\mathrm{u}} P^{\mathrm{u}}$ and $P^{\mathrm{u}} S^{\mathrm{d}}$ represent the reflected and transmitted waves generated by the upward incident P-wave in the solid layer; and $S^{\mathrm{u}} P^{\mathrm{d}}, S^{\mathrm{u}} P^{\mathrm{u}}$ and $S^{\mathrm{u}} S^{\mathrm{d}}$ represent the reflected and transmitted waves generated by the upward incident S-wave in the solid layer.

\section{Analytical expression of coefficients}

According to Eq. (11), we obtain five reflection coefficients and four transmission coefficients at the submarine interface. Considering the demand for seafloor seismic exploration, $P^{\mathrm{d}} P^{\mathrm{u}}, P^{\mathrm{d}} P^{\mathrm{d}}$ and $P^{\mathrm{d}} S^{\mathrm{d}}$ in Eq. (11) were studied separately to obtain the analytical understanding of the reflection and transmission coefficient of the downward incident P-wave. Thus, $P^{\mathrm{d}} P^{\mathrm{u}}, P^{\mathrm{d}} P^{\mathrm{d}}$ and $P^{\mathrm{d}} S^{\mathrm{d}}$ are renamed as $R_{P P}, T_{P P}$ and $T_{P S}$ to facilitate the presentation. With the intermediate variables in Eq. (10), the reflection and transmission coefficients of the downward incident P-wave at the submarine interface can be expressed in fractional forms: 


$$
\left\{\begin{aligned}
R_{P P} & =\frac{b \cos i_{1}+a \cos i_{2}}{b \cos i_{1}-a \cos i_{2}} \\
T_{P P} & =\frac{2 \cos i_{1}(c-a)}{b \cos i_{1}-a \cos i_{2}} \\
T_{P S} & =\frac{2 \rho_{1} \alpha_{1} d \cos i_{1}}{b \cos i_{1}-a \cos i_{2}}
\end{aligned}\right.
$$

In Eq. (12), $R_{P P}, T_{P P}$ and $T_{P S}$ must be normalized to ensure that the flux of energy leaving the boundary equal that in the incident wave (Kennett 1979; Aki and Richards 1980). Therefore, with a normalization factor, the complete form of Eq. (12) can be obtained as follows:

$$
\left\{\begin{array}{c}
R_{P P}=\frac{b \cos i_{1}+a \cos i_{2}}{b \cos i_{1}-a \cos i_{2}} \\
T_{P P}=\frac{2 \cos i_{1}(c-a)}{b \cos i_{1}-a \cos i_{2}} \sqrt{\frac{\rho_{2} \alpha_{2} \cos i_{2}}{\rho_{1} \alpha_{1} \cos i_{1}}} \\
T_{P S}=\frac{2 \rho_{1} \alpha_{1} d \cos i_{1}}{b \cos i_{1}-a \cos i_{2}} \sqrt{\frac{\rho_{2} \beta_{2} \cos j_{2}}{\rho_{1} \alpha_{1} \cos i_{1}}}
\end{array}\right.
$$

In Eq. (13), the $R_{P P}, T_{P P}$ and $T_{P S}$ have the same denominator in fractional form. We assume that the wave impedance of the incidence P-wave in the upper layer is $I_{1}$, the wave impedance of the transmission $\mathrm{P}$-wave in the seabed layer is $I_{2}$, and the wave impedance of the transmission Swave in the seabed layer is $I_{3}$; thus, Eq. (13) can be expressed in the form of wave impedance on both sides of the interface as follows:

$$
\left\{\begin{array}{c}
R_{P P}=1+\frac{2 I_{1} \cos i_{2}}{G} \\
T_{P P}=\frac{-2 I_{1} \cos i_{1} \cos 2 j_{2}}{G} \sqrt{\frac{I_{2} \cos i_{2}}{I_{1} \cos i_{1}}} \\
T_{P S}=\frac{4 I_{1} \sin j_{2} \cos i_{1} \cos i_{2}}{G} \sqrt{\frac{I_{3} \cos j_{2}}{I_{1} \cos i_{1}}}
\end{array}\right.
$$

where $I_{1}=\rho_{1} \alpha_{1}, I_{2}=\rho_{2} \alpha_{2}$ and $I_{3}=\rho_{2} \beta_{2}$. The common variable $G$ denotes the relation between the reflection coefficient and transmission coefficient of the submarine interface as a common denominator, and the form of $G$ is as follows:

$$
\begin{aligned}
G= & 2 \sin j_{2} \sin 2 j_{2} \cos i_{1}\left(I_{2} \cos j_{2}-I_{3} \cos i_{2}\right)-I_{1} \cos i_{2} \\
& -I_{2} \cos i_{1}
\end{aligned}
$$

In Eq. (15), $G$ plays an important role in the analytical analysis and study of the seabed reflection and transmission coefficient. From the common variable $G$, we observe that the size of the reflection and transmission coefficient is simply affected by the difference in the wave impedance on both sides of the interface and is closely related to the incident angle $i_{1}$ and the two transmission angles $i_{2}$ and $j_{2}$. The common variable $G$ associates the reflection and transmission coefficients at the submarine interface, which makes it possible to analyse the reflection and transmission coefficients. According to Snell's law in Eq. (2), the relationship between these three angles is as follows:

$\sin i_{1}=\frac{\alpha_{1}}{\alpha_{2}} \sin i_{2}=\frac{\alpha_{1}}{\beta_{2}} \sin j_{2}$

Therefore, when the incident angle $i_{1}$ changes, whether the ratios $\frac{\alpha_{1}}{\alpha_{2}}$ and $\frac{\alpha_{1}}{\beta_{2}}$ are larger than 1 or not will have different effects on the transmission angles $i_{2}$ and $j_{2}$. In a stiff seabed, the $\alpha_{2}$ and $\beta_{2}$ are generally greater than the seawater velocity, thus, $\frac{\alpha_{1}}{\alpha_{2}}<1$ and $\frac{\alpha_{1}}{\beta_{2}}<1$. If the incident angle $i_{1}$ increases to a critical extent, $\sin i_{2}$ and $\sin j_{2}$ will appear to be greater than 1 , in which case $\cos i_{2}$ and $\cos j_{2}$ become purely imaginary. Since $\cos i_{2}$ and $\cos j_{2}$ denote the $k_{z}$ of the $\mathrm{P}$ - and $\mathrm{S}$-waves in the seabed layer, respectively, the transmitted waves become an inhomogeneous wave whose amplitude exponentially decays with distance $\mathrm{z}$ away from the submarine interface. At this time, it seems meaningless to discuss the transmission coefficient of the P- and Swaves in the stiff seabed. Separately, while in a soft seabed layer, $\beta_{2}$ of the seabed is usually lower than the velocity of the sea water, and there is no case of $\frac{\alpha_{1}}{\beta_{2}}<1$. Thus, the establishment of Eq. (12) requires a certain range of conditions, which ensures that the transmitted $\mathrm{P}$ - and S-waves do not become inhomogeneous waves and propagate along the seafloor interface in the wide-angle reflection range. Therefore, Eq. (14) can be further expressed as follows:

$$
\begin{cases}R_{P P}=1+\frac{2 I_{1} \cos i_{2}}{G}, & \sin i_{1} \leq 1 \\ T_{P P}=\frac{-2 I_{1} \cos i_{1} \cos 2 j_{2}}{G} \sqrt{\frac{I_{2} \cos i_{2}}{I_{1} \cos i_{1}}}, & \sin i_{1} \leq \frac{\alpha_{1}}{\alpha_{2}} \\ T_{P S}=\frac{4 I_{1} \sin j_{2} \cos i_{1} \cos i_{2}}{G} \sqrt{\frac{I_{3} \cos j_{2}}{I_{1} \cos i_{1}}}, & \sin i_{1} \leq \frac{\alpha_{1}}{\beta_{2}}\end{cases}
$$

\section{Discussions of critical angles}

Equation (17) is the reflection and transmission coefficient of the downward incident P-wave at the submarine interface. As seen in Eq. (17), both the reflection and transmission coefficients are mainly affected by wave impedance $I$, incident angle $i_{1}$ and transmission angles $i_{2}$ and $j_{2}$ at the submarine interface. The change in the reflection and transmission coefficient with the incident angle, which is a nonlinear problem, appears to be quite complex. However, when the incident angle reaches a critical size, the transmission angles $i_{2}$ and $j_{2}$ in Eq. (16) reach $90^{\circ}$, and the amplitude value and phase angle of the reflection and transmission coefficient will show a special change in these incident angles. The incident angle of the $\mathrm{P}$-wave at this time is called the critical angle. 
With different media properties of the seafloor interface, critical angles in the submarine interface can be divided into two categories: the P-wave critical angle and the converted $\mathrm{S}$-wave critical angle. Parameters $c_{P}$ and $c_{S}$ are used to represent the P-wave critical angle and the converted $\mathrm{S}$-wave critical angle, respectively. The reflection coefficients of the submarine interface vary with the incident angle in a "segmented" form with two types of critical angles as the dividing point. Different submarine interfaces, such as stiff and soft seabed, will generate different types of critical angles. From Eq. (16), we know that there will be $c_{P}$ and $c_{S}$ in the stiff seabed, whereas in the soft seabed, only $c_{P}$ exists. Therefore, the reflection coefficient of the stiff submarine interface shows a "three-stage" characteristic with A change in the incident angle, whereas the soft seabed presents "two stages."

According to Eq. (17), these submarine critical angles can be discussed as follows:

1. At $c_{P}, i_{2}=90^{\circ}, \sin i_{2}=1$, the incident angle satisfies $\sin i_{1}=\frac{\alpha_{1}}{\alpha_{2}}, \quad$ and thus, $\quad \cos i_{2}=0 \quad$ and $G=-I_{2} \cos i_{1} \cos ^{2}\left(2 j_{2}\right)$. Placing these parameters into Eq. (17), we obtain $R_{P P}=1, T_{P P}=0$ and $T_{P S}=0$. When the incidence angle $i_{1}$ is greater than $c_{P}$, the transmitted $\mathrm{P}$-wave becomes an inhomogeneous wave.

2. At $c_{S}, j_{2}=90^{\circ}, \sin j_{2}=1$, the incident angle satisfies $\sin i_{1}=\frac{\alpha_{1}}{\beta_{2}}, \quad$ and thus, $\cos j_{2}=0 \quad$ and $G=-\left(I_{1} \cos i_{2}+I_{2} \cos i_{1}\right)$. With Eq. (17), we obtain $\left|R_{P P}\right|=1$ and $T_{P S}=0$ because $\cos i_{2}$ becomes purely imaginary at this critical angle. When the incidence angle is greater than $c_{S}$, the converted $\mathrm{S}$-wave becomes an inhomogeneous wave.

Therefore, we see that the three stages of the reflection coefficient changing with the incident angle of the stiff submarine interface are $0^{\circ}$ to $c_{P}, c_{P}$ to $c_{S}$ and $c_{S}$ to $90^{\circ}$. In the first stage of $0^{\circ}$ to $c_{P}$, the amplitude value of the reflection coefficient is a positive real number with $G<0$ and gradually increases to 1 when the incident angle reaches the $\mathrm{P}$-wave critical angle. In the second stage between $c_{P}$ and $c_{S}$, the amplitude of the reflection coefficient becomes an imaginary number because the wave number of the P-wave and converted $\mathrm{S}$-wave in the $\mathrm{z}$ direction becomes purely imaginary. In the third stage of $c_{S}$ to $90^{\circ}$, the amplitude of reflection coefficient satisfies the following:

$R_{P P}=\frac{I_{2} \cos i_{1}-I_{1} \cos i_{2}}{I_{2} \cos i_{1}+I_{1} \cos i_{2}}$

where the numerator and denominator of $R_{P P}$ in Eq. (18) are a pair of conjugate complex numbers, and thus, in this stage, the absolute value of $R_{P P}$ is always 1 when the incident angle varies. At the soft submarine interface, the third stage does not exist because the shear wave velocity is small and there is no $c_{S}$ angle. Riedel and Theilen (2001) have found that the size of the critical angle in the shallow marine sediments was mainly affected by the P-wave velocity compared with the $\mathrm{S}$-wave velocity and density, which can be further explained by our conclusion that there is only a P-wave critical angle in the soft seabed. Thus, the study of the submarine critical angles is the key to obtain the characteristics of the submarine reflection and transmission coefficient, which can aid us in recognizing the seabed layer in a different medium.

Therefore, the amplitude of the reflection and transmission coefficient at the critical angles shows a clear characteristic, which can be easily recognized in the curve of the reflection and transmission coefficient with the incidence angle. The size and type of the critical angles are affected by the P- and S-wave velocities of the solid seabed layer. It is the critical angle that associates the properties of the submarine medium with the seafloor coefficient characteristics. On the one hand, if we know the submarine medium properties, we can infer the characteristics of the seabed reflection and transmission coefficient. On the other hand, if we obtain the seabed reflection and transmission coefficient from the ocean seismic data, we can invert the velocity of the seafloor interface.

\section{Synthetic model study}

To validate our conclusion, synthetic models of both the stiff and soft seabed are established in this section as examples for simulating the reflection and transmission coefficients at the submarine interface. The model parameters of the stiff seafloor interface are shown in Table 2. At the stiff seafloor interface, the P-wave velocity $\alpha_{1}$ and density $\rho_{1}$ in the seawater layer are set to $1490 \mathrm{~m} / \mathrm{s}$ and $1025 \mathrm{~kg} / \mathrm{m}^{3}$, respectively; the P-wave velocity $\alpha_{2}, \mathrm{~S}$-wave velocity $\beta_{2}$ and density $\rho_{2}$ in the solid seabed layer are set to $4000 \mathrm{~m} / \mathrm{s}, 1920 \mathrm{~m} / \mathrm{s}$ and $2460 \mathrm{~kg} / \mathrm{m}^{3}$, respectively. Therefore, the P-wave velocity and S-wave velocity of the seabed layer are greater than the velocity of the sea water, and thus, $c_{P}$ and $c_{S}$ must be in the reflection coefficient curves with the incident angle. With Eq. (17), we can calculate the reflection and transmission coefficient of the stiff seabed with the parameters given in Table 2. The calculation results are shown in Fig. 2.

Table 2 Model parameters at the stiff seafloor interface

\begin{tabular}{llll}
\hline Layer & $\rho\left(\mathrm{kg} / \mathrm{m}^{3}\right)$ & $\alpha(\mathrm{m} / \mathrm{s})$ & $\beta(\mathrm{m} / \mathrm{s})$ \\
\hline Seawater layer & 1025 & 1490 & - \\
Seabed layer & 2460 & 4000 & 1920 \\
\hline
\end{tabular}




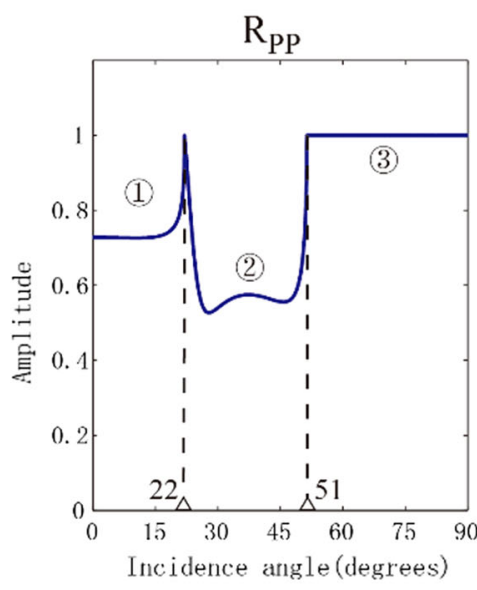

(a)

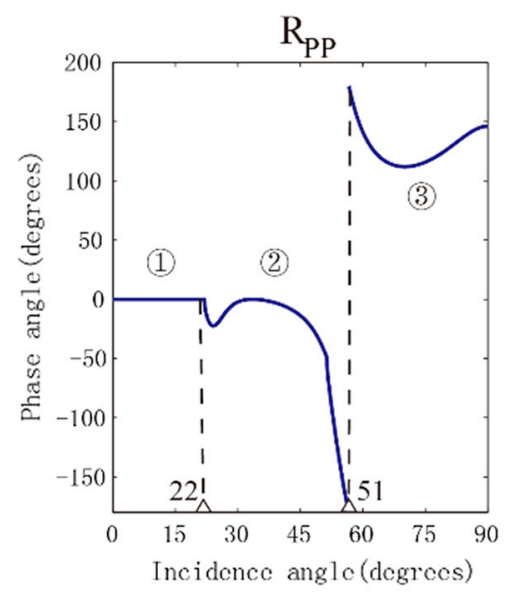

(d)

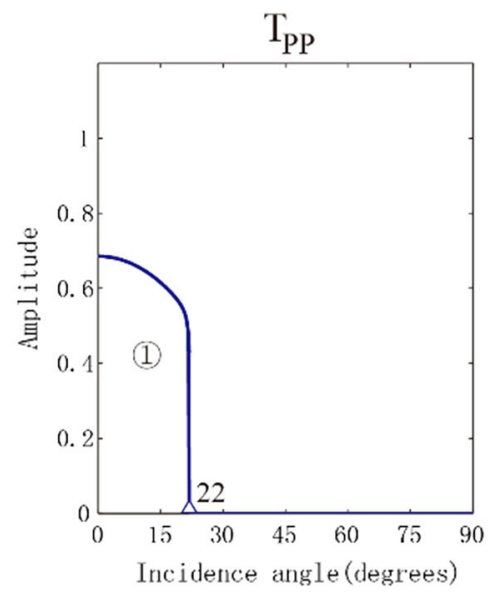

(b)

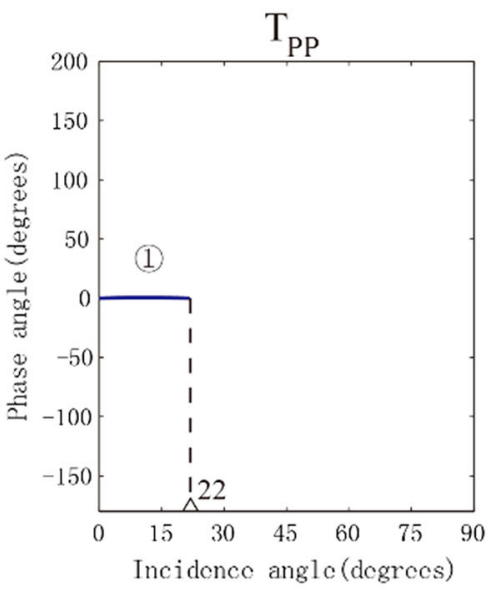

(e)

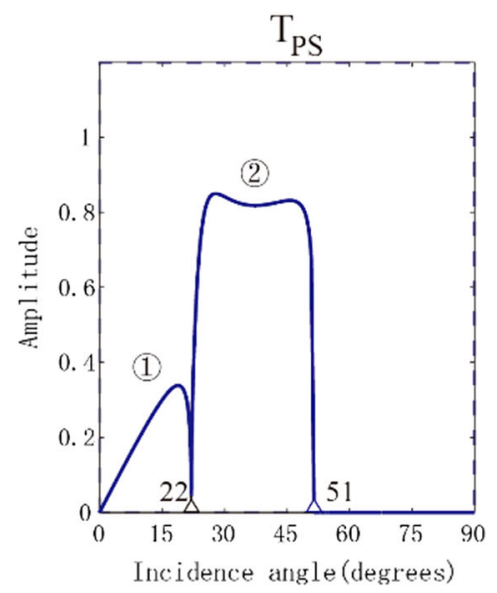

(c)

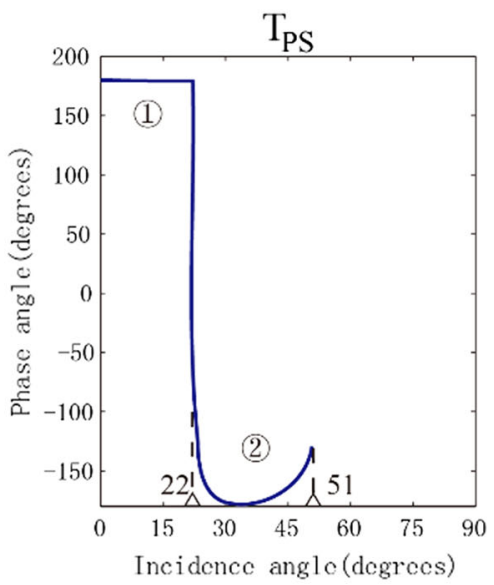

(f)
Fig. 2 Reflection and transmission coefficient in the stiff interface model. a-c shows the change in the amplitude of the reflection and transmission coefficient with the incidence angle, and $\mathbf{d}-\mathbf{f}$ shows the change in the corresponding phase angle with the incident angle. In the seawater layer, $\rho_{1}=1025 \mathrm{~kg} / \mathrm{m}^{3}, \alpha_{1}=1490 \mathrm{~m} / \mathrm{s}$; in the seabed

Figure $2 \mathrm{a}-\mathrm{c}$ shows the amplitude change of the reflection and transmission coefficient with the incident angle. From Fig. 2a, the amplitude value of the reflection coefficient $R_{P P}$ reaches 1 at the two incident angles of $22^{\circ}$ and $51^{\circ}$ and remain unchanged since the incidence angle is greater than $51^{\circ}$. According to the previous discussion, $22^{\circ}$ and $51^{\circ}$ are $c_{P}$ and $c_{S}$, respectively. When the incidence angle reaches the critical angle, the transmission angle of the $\mathrm{P}$-wave and converted $\mathrm{S}$-wave in the seabed layer reaches $90^{\circ}$; when the incidence angle is greater than the critical angle, the inhomogeneous surface waves propagating along the interface will be generated, and thus, the amplitude of the transmission coefficient $T_{P P}$ and $T_{P S}$ in Fig. $2 \mathrm{~b}$, c turns into zero at $c_{P}$ and $c_{S}$, respectively. Given the energy normalization in the submarine interface, the amplitude value of $R_{P P}, T_{P P}$ and $T_{P S}$ at the same incident angle satisfies the following: layer, $\rho_{2}=2460 \mathrm{~kg} / \mathrm{m}^{3}, \quad \alpha_{2}=4000 \mathrm{~m} / \mathrm{s}$ and $\beta_{2}=1920 \mathrm{~m} / \mathrm{s}$. The angles in the abscissa marked with " $\triangle$ " represent the critical angles in this stiff seafloor interface model. (1), (2) and (3) represent the three stages of the coefficient in the variation with the incident angle

$\left|R_{P P}\right|^{2}+\left|T_{P P}\right|^{2}+\left|T_{P S}\right|^{2}=1$

Figure $2 \mathrm{~d}-\mathrm{f}$ shows the phase variation with the incident angle. In Fig. $2 \mathrm{~d}$, the phase angle of $R_{P P}$ shows a significant change at the two critical angles: at $c_{P}$, the phase angle varies from $0^{\circ}$ to a negative angle; at $c_{S}$, the phase angle of the reflection coefficient is reversed from $-180^{\circ}$ to $180^{\circ}$. Similar to amplitude variation, the phase variation with the incident angle of $R_{P P}$ also presents a "segmented" form with two critical angles as the dividing point. In Fig. 2e, the phase angle of $T_{P P}$ remains at $0^{\circ}$ in the stage $0^{\circ}$ to $c_{P}$, and the amplitude value of $T_{P P}$ is always real. In Fig. 2f, there is a significant phase change in $T_{P S}$ at angle $c_{S}$, where the amplitude value of $T_{P S}$ turns from real to imaginary at $c_{S}$. With the phase variation with the angle (PVA) of the reflection and transmission coefficient, the elastic inversion at near and post-critical angles can be used to obtain the 
parameters at the submarine interface (Rafipour and Herrin 1986; Zhu and McMechan 2012). Therefore, the analytical study of both amplitude and phase variation with the incident angle is the basis of the velocity and density inversion at the seafloor interface.

In the case of the stiff seabed, we can see two critical angles as the dividing points in the curve of the reflection coefficient with the incidence angle. The amplitude value and phase angle of the reflection coefficient $R_{P P}$ change obviously at the two critical angles, and the curve shows the special "three-stage" change in the stiff seafloor interface. The transmission coefficient $T_{P P}$ decreases to zero at $c_{P}$ in the first stage, and then, the transmission $\mathrm{P}$ wave becomes an inhomogeneous surface wave in the second stage. As the incident angle increases further in the third stage, $T_{P S}$ is zero at $c_{S}$, and the converted $\mathrm{S}$-wave also becomes an inhomogeneous surface wave propagating along the seafloor interface. The determination of the two critical angles of the stiff seabed interface during marine seismic exploration can determine the reflection and transmission coefficient characteristics of the incident P-wave, which is very important for the calculation and interpretation of the reflection and transmission at the submarine interface.

Similarly, we have also established a soft submarine model, and the parameters are shown in Table 3. At the soft seafloor interface, the P-wave velocity $\alpha_{1}$ and density $\rho_{1}$ of the seawater layer are set to $1490 \mathrm{~m} / \mathrm{s}$ and $1025 \mathrm{~kg} /$ $\mathrm{m}^{3}$, respectively, which are same as the stiff model. The Pwave velocity $\alpha_{2}$, S-wave velocity $\beta_{2}$ and density $\rho_{2}$ of the seabed layer are $1800 \mathrm{~m} / \mathrm{s}, 522 \mathrm{~m} / \mathrm{s}$ and $1600 \mathrm{~kg} / \mathrm{m}^{3}$, respectively, which are all lower than those of the stiff interface. The P-wave velocity of the seabed layer in this model is greater than the seawater velocity, while the Swave velocity of the seabed layer is smaller than the seawater layer, and thus, there is only $c_{P}$ in the reflection coefficient curves with the incident angle at the soft submarine interface. With Eq. (17), we can calculate the reflection and transmission coefficient of the soft seabed with the parameters provided in Table 3. The calculation results are shown in Fig. 3.

Figure $3 \mathrm{a}-\mathrm{c}$ shows the amplitude change of the reflection and transmission coefficient with the incident angle, and $(\mathrm{d}-\mathrm{f})$ shows the change in the corresponding phase angle with the incident angle, respectively. From Fig. 3a, the amplitude value of the reflection coefficient $R_{P P}$ reaches 1 at the incident angle $56^{\circ}$, which is the $\mathrm{P}$-wave

Table 3 Model parameters at the soft seafloor interface

\begin{tabular}{llll}
\hline Layer & $\rho\left(\mathrm{kg} / \mathrm{m}^{3}\right)$ & $\alpha(\mathrm{m} / \mathrm{s})$ & $\beta(\mathrm{m} / \mathrm{s})$ \\
\hline Seawater layer & 1025 & 1490 & - \\
Seabed layer & 1600 & 1800 & 522 \\
\hline
\end{tabular}

critical angle $c_{P}$. As the incidence angle reaches $c_{P}$, the transmission angle of the P-wave in the seabed layer reaches $90^{\circ}$; when the incidence angle is greater than the critical angle, the P-waves turn into inhomogeneous waves propagating along the interface, and thus, the amplitude of the transmission coefficient $T_{P P}$ in Fig. $2 \mathrm{~b}$ turns into zero at $c_{P}$. Since the $\mathrm{S}$-wave critical angle does not exist at the soft submarine interface, the transmission coefficient $T_{P S}$ exists in the full incidence angle range. In Fig. $2 \mathrm{~d}$, the phase angle of $R_{P P}$ shows a significant change at $c_{P}$ from $0^{\circ}$ to a negative angle. In Fig. $2 \mathrm{e}$, the phase angle of $T_{P P}$ remains at $0^{\circ}$ in the stage of $0^{\circ}$ to $56^{\circ}$, and the amplitude value of $T_{P P}$ is always real. In Fig. $2 \mathrm{f}$, there is a significant phase change in $T_{P S}$ at angle $c_{S}$. Therefore, in the case of the soft seabed, we can only see a larger P-wave critical angle as a dividing point in the curve of the reflection and transmission coefficient with the incidence angle. The amplitude and phase angle of $R_{P P}$ and transmission coefficient $T_{P S}$ change obviously at the two critical angles, and the curve shows the special "two-stage" change at the soft seafloor interface.

\section{Real seismic data comparison: Gulf of Mexico}

To further verify the accuracy of our analytical results, we compared our synthetic results with the real seismic data chosen from the Gulf of Mexico dataset. Therefore, in this section, we first present a simplified isotropic horizontal layered model for the Gulf of Mexico seabed, which has a high-velocity, thin layer beneath the consolidated sediment and water layers. The parameters of the seafloor model are shown in Table 4. As shown in Table 4, the model is divided into six horizontal layers, each of which has a different speed, density and thickness. Among the layers, the first is the water layer, the second is the sedimentary layer, the third is a high-speed thin layer, and the other layers under these layers are solid layers. The thickness of the water layer is $500 \mathrm{~m}$, whereas the depth and thickness of the high-speed thin layer are 650 and $150 \mathrm{~m}$. The water gun and receivers are both placed $10 \mathrm{~m}$ below the water surface in the synthetic simulation, and the sine wave at $25 \mathrm{~Hz}$ is set as the source function. The minimum offset is $200 \mathrm{~m}$, the interval between each receiver is $100 \mathrm{~m}$, and there are 45 receivers in total (the maximum one-sided offset is $4700 \mathrm{~m}$ ), which is the same as the real data observation system. Using the analytical expression of reflection and transmission coefficient in Eq. (17), we can calculate the travel time and amplitude of the different wave types in this model.

Since the reflection and transmission of the seafloor interface are submerged in multiple records of different 


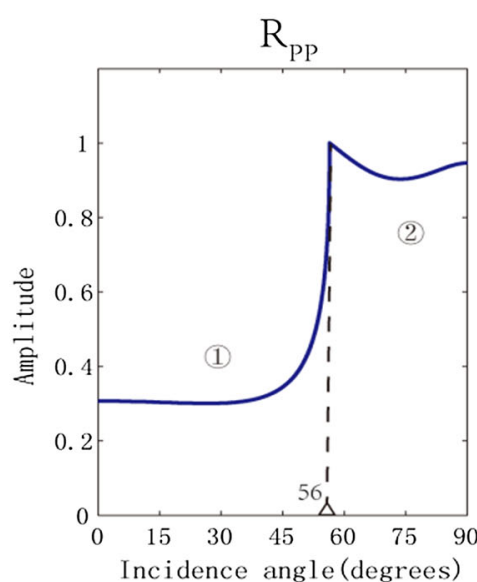

(a)

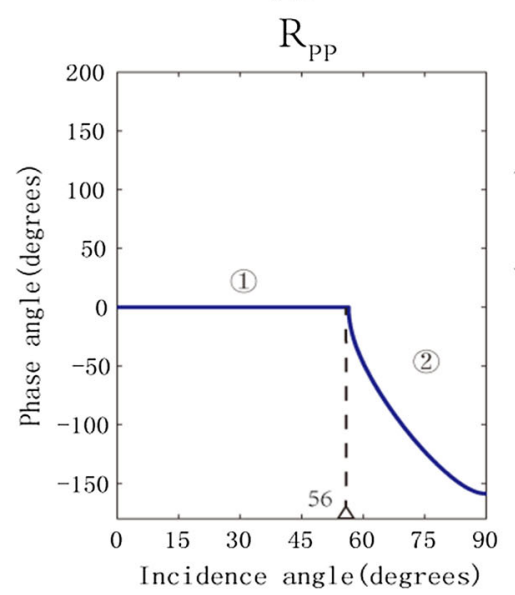

(d)

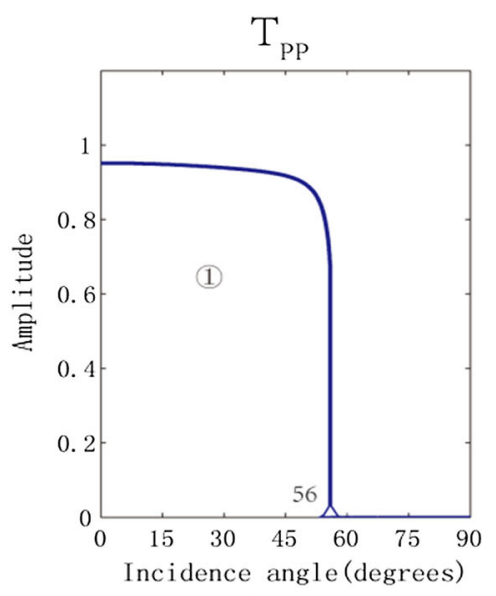

(b)

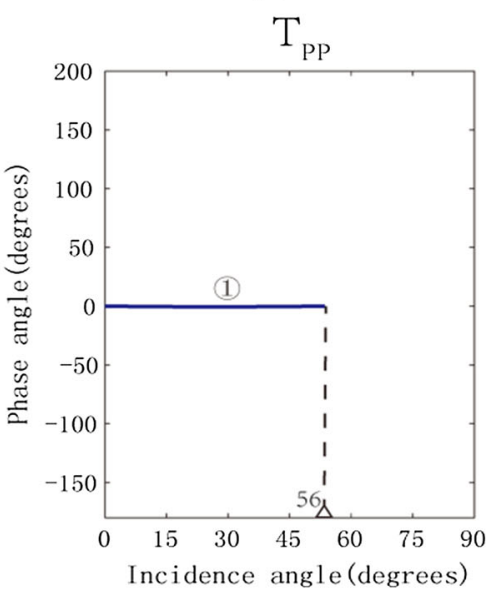

(e)

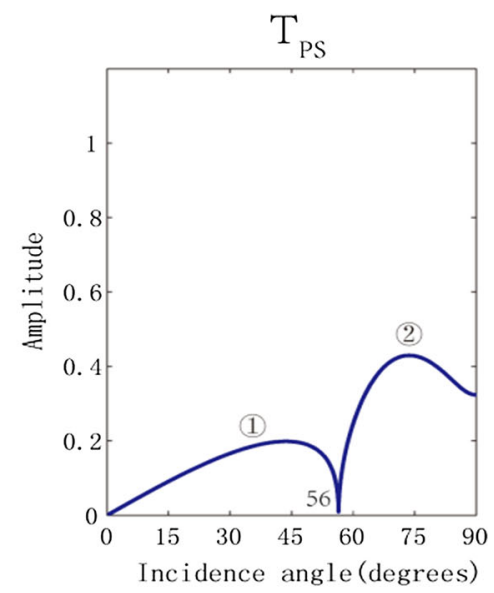

(c)

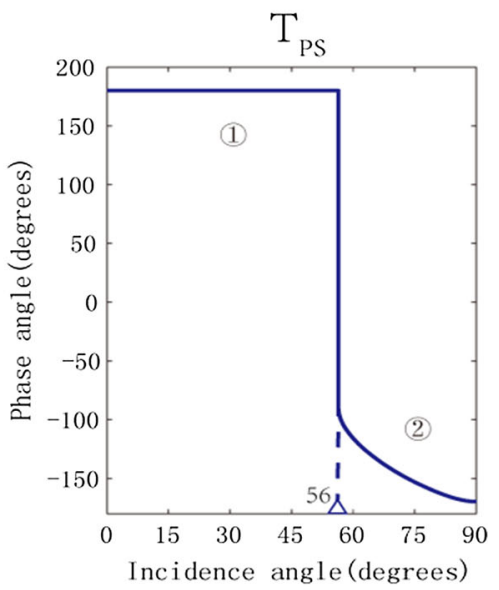

(f)
Fig. 3 Reflection and transmission coefficient at the soft interface model. a-c shows the change in the amplitude of the reflection and transmission coefficient with the incidence angle, respectively, and df shows the change in the corresponding phase angle with the incident angle, respectively. In the seawater layer, $\rho_{1}=1025 \mathrm{~kg} / \mathrm{m}^{3}$ and

interfaces, it is very difficult to directly study the reflection and transmission of the seafloor interface in the real data. The amplitude characteristics of inhomogeneous waves propagating in a high-speed, thin layer are the most direct evidence we have to verify the accuracy of our results. Based on the previous analytical discussion, we can further obtain the propagation mechanism of the inhomogeneous waves in the high-speed, thin layer. As shown in Fig. 4, the P-wave in the water layer transforms into a downward homogeneous S-wave at the liquid-solid boundary. The homogeneous S-wave enters the high-velocity thin layer at the critical angle and propagates horizontally in the layer as the inhomogeneous wave does when the incident angle is larger than the critical angle, which is similar to our previous analysis of the reflection and transmission coefficients at larger incidence angles. Then, the inhomogeneous wave is converted into the upward homogeneous S-wave at $\alpha_{1}=1490 \mathrm{~m} / \mathrm{s} ; \quad$ in the seabed layer, $\rho_{2}=1600 \mathrm{~kg} / \mathrm{m}^{3}, \quad \alpha_{2}=$ $1800 \mathrm{~m} / \mathrm{s}$ and $\beta_{2}=522 \mathrm{~m} / \mathrm{s}$. The angles in the abscissa marked with " $\triangle$ " represent the critical angles in this stiff seafloor interface model. (1) and (2) represent the two stages of the coefficient in the variation with the incident angle, respectively

the same critical angle and finally changes into a P-wave in the water layer at the solid-liquid interface.

In this model, the sedimentary layer is under the stiff seafloor, as the $\mathrm{S}$-wave velocity is larger than the $\mathrm{P}$-wave velocity in the water layer. This is because the velocity and density of the stiff seafloor are greater than those of the soft seafloor, which ensures that the inhomogeneous wave has enough energy when propagating as an S-wave beneath the water layer. Therefore, the stiff seafloor becomes a strong source of transverse waves in the multiple conversion between the $\mathrm{P}$ - and S-waves. In contrast, the P- and S-wave velocities and densities of the sediments beneath the soft seafloor aquifers are relatively smaller, and the conversion between the P- and S-waves decreases at the solid-liquid interface. An $\mathrm{S}$-wave with enough energy is difficult to generate at the solid-liquid interface, and thus, the energy conversion into a high-speed, thin layer is weak, which attenuates the inhomogeneous wave or even disappears. 
Table 4 The seafloor model of isotropic horizontal layered media

\begin{tabular}{lllll}
\hline Layer & P-velocity $\alpha(\mathrm{km} / \mathrm{s})$ & S-velocity $\beta(\mathrm{km} / \mathrm{s})$ & Density $\rho\left(\mathrm{g} / \mathrm{cm}^{3}\right)$ & Thickness $h(\mathrm{~km})$ \\
\hline 1 & 1.5 & 0.0 & 1.0 & 0.5 \\
2 & 3.5 & 2.0 & 2.0 & 0.2 \\
3 & 5.8 & 3.4 & 2.6 & 0.15 \\
4 & 3.6 & 2.1 & 2.0 & 0.2 \\
5 & 4.0 & 2.3 & 2.45 & 0.3 \\
6 & 5.8 & 3.4 & 2.6 & $\propto$ \\
\hline
\end{tabular}

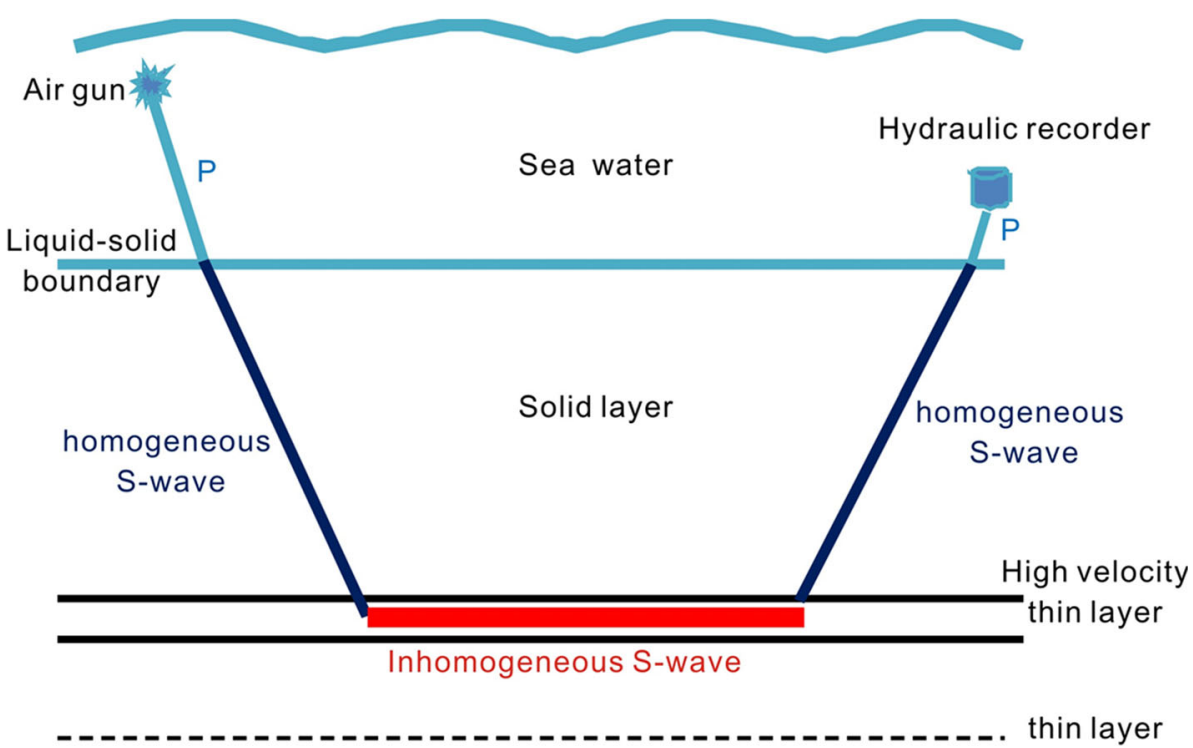

Fig. 4 Mechanism of inhomogeneous waves in the seafloor with a thin, highvelocity layer
With the reflection and transmission coefficient expression of the liquid-solid interface, solid-solid interface and solid-liquid interface, we can calculate the theoretical seismograms of different types of waves in this model. The results are shown in Fig. 5a, and the real data that were used are shown in Fig. $5 b$ for the sake of comparison. Despite ignoring details of the horizontal velocity structure, the characteristics of the travel time and amplitude variations obtained using our model are highly similar to the real data from the Gulf of Mexico. On the one hand, the travel time and amplitude of the different order multiples are similar to the actual data. Multiples reflected with an incidence angle close to the P-wave critical angle or greater than the S-wave critical angle are seen as large amplitude waves, which is consistent with the variation in the reflection coefficient at the stiff seafloor interface, which we previously obtained. On the other hand, we successfully simulated a linear time-distance curve for the Gulf of Mexico data (seen in Fig. 5), which is similar to the refraction wave but has a higher velocity. This curve provides a more reasonable explanation from the perspective of inhomogeneous waves by establishing a more reasonable model and reveals the existence of high-speed, thin layers under the stiff seafloor in the Gulf of Mexico.
Simultaneously, this curve also verifies the accuracy of our reflection and transmission coefficient calculation.

\section{Conclusions}

In this paper, we mainly focused on the analytical study of the reflection and transmission coefficient at the submarine interface. According to the boundary conditions at the submarine interface, we have obtained the analytical expression, which is applicable to the analytical study. The analytical expressions of the seafloor coefficient generated by the downward incidence P-wave are obtained using the energy normalization at the seafloor interface. With the final form of the analytic expression, the reflection and transmission coefficients of the seafloor using different parameters are discussed. The change in the reflection and transmission coefficients with the incidence angle presents a "segmented" characteristic with the critical angles as the dividing points. The size and type of critical angles are affected by the velocities of the $\mathrm{P}$ - and $\mathrm{S}$-waves in the seabed layer. At critical angles, the amplitude value and phase angle of the reflection and transmission coefficients can vary significantly. Different $\mathrm{P}$ - and $\mathrm{S}$-wave velocities at 


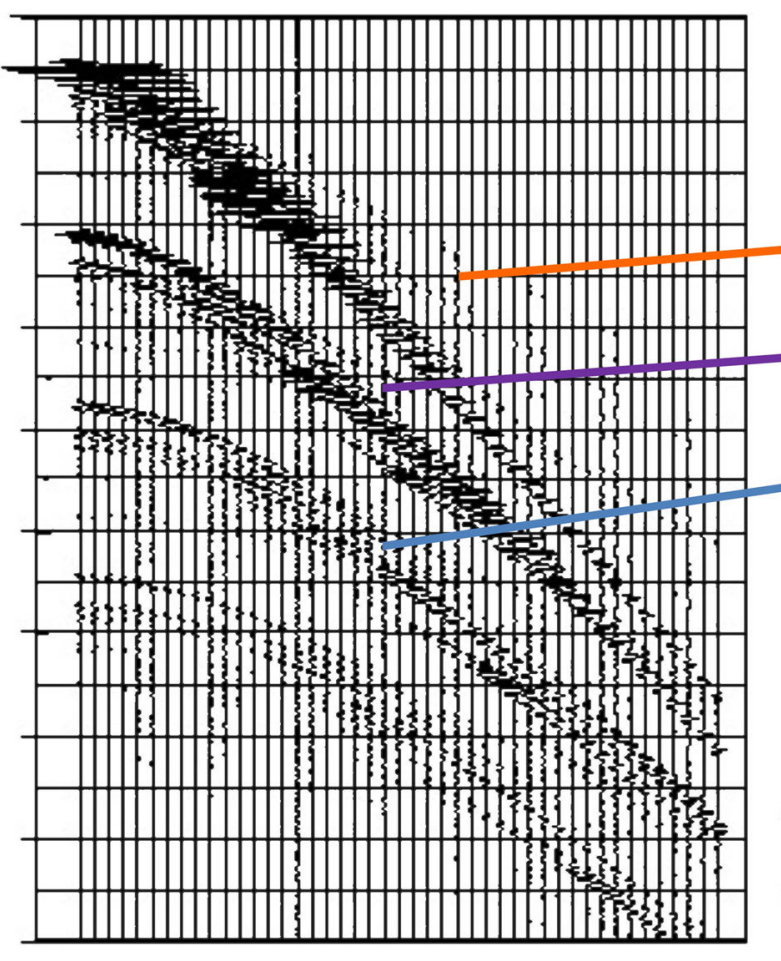

Synthetics

(a)

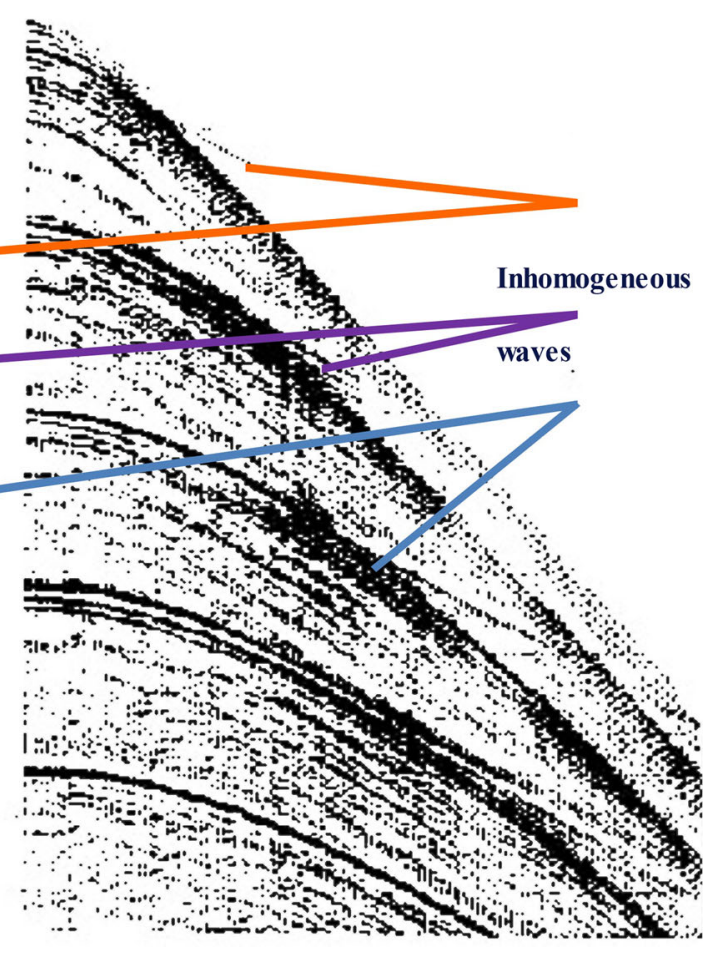

Real data

(b)

Fig. 5 Synthetics and the actual hydraulic record from the high-speed, thin layer

different seabed layer interfaces can lead to different submarine critical angle types and sizes. With the analytical solution, we know that there are two critical angles in the stiff seabed, whereas in the soft seabed, there is only one larger P-wave critical angle due to the low velocity of the $\mathrm{S}$-wave. Therefore, the reflection coefficient of the stiff submarine interface shows a "three-stage" characteristic with the change in incident angle, whereas the soft seabed presents a "two-stage" characteristic. In each stage, the reflection and transmission coefficients show different variation characteristics with the incident angle. Therefore, it seems reasonable to determine the critical angle type by its characteristics in the reflection and transmission curve, and then, the properties of the submarine interface can be determined.

To validate our conclusions from the analytical study, we first established two typical seafloor models to find the similarities in the synthetic results. The reflection and transmission coefficients in both the stiff and soft seafloor models show the same characteristic, which we have previously discussed. Then, we extended our models and calculated the theoretical seismograms of different waves in this model, and the travel time and amplitude variations obtained with our model are highly similar to the real data from the Gulf of Mexico. In the future, we will further expand the complexity of our model, with the aim to gain better understanding and correspondence in the actual data.

Acknowledgements This research was funded by the National Natural Science Foundation of China (Grant Nos. 41590862 and 41174045) and independent technology development projects from the Institute of Geology, China Earthquake Administration. We would like to thank senior engineer Guangming $\mathrm{Yu}$ of the Institute of Multicomponent Seismic Technology in Beijing for assistance with seismic data processing.

Open Access This article is distributed under the terms of the Creative Commons Attribution 4.0 International License (http://creative commons.org/licenses/by/4.0/), which permits unrestricted use, distribution, and reproduction in any medium, provided you give appropriate credit to the original author(s) and the source, provide a link to the Creative Commons license, and indicate if changes were made.

\section{References}

Aki K, Richards PG (1980) Quantitative seismology: theory and methods. W.H. Freeman \& Co, New York

Aleardi M, Tognarelli A (2016) The limits of narrow and wide-angle AVA inversions for high $\mathrm{Vp} / \mathrm{Vs}$, ratios: an application to elastic seabed characterization. J Appl Geophys 131:54-68

Alemie W, Sacchi MD (2011) High-resolution three-term AVO inversion by means of a Trivariate Cauchy probability distribution. Geophysics 76(3):R43-R55 
Bortfeld R (1961) Approximation to the reflection and transmission coefficients of plane longitudinal and transverse waves. Geophys Prospect 9:485-502

Bull JM, Quinn R, Dix JK (1998) Reflection coefficient calculation from marine high resolution seismic reflection (Chirp) Data and Application to an Archaeological Case Study. Mar Geophys Res 20(1):1-11

Carcione J (2001) Wave fields in real media: theory and numerical simulation of wave propagation in anisotropic anelastic and porous media. Pergamon Press 6:187-193

Carcione J, Helle H (2004) The physics and simulation of wave propagation at the ocean bottom. Geophysics 69(3):825-839

Castagna JP, Backus MM (1993) Offset-dependent reflectivitytheory and practice of AVO analysis. SEG

Crampin S (1981) Review of wave motion in anisotropic and cracked media. Wave Motion 3(4):343-391

Das S, Bora SN (2014) Reflection of oblique ocean water waves by a vertical porous structure placed on a multi-step impermeable bottom. Appl Ocean Res 47(47):373-385

Downton JE, Ursenbach C (2006) Linearized amplitude variation with offset (AVO) inversion with supercritical angles. Geophysics 71(5):49-55

Feng SJ, Chen ZL, Chen HX (2016) Reflection and transmission of plane waves at an interface of water/multilayered porous sediment overlying solid substrate. Ocean Eng 126:217-231

Gu HM, Wang JY, Zhu GM (2002) Calculation of reflection coefficient in frequency-wave-number domain using seafloor seismic multi-component data. Chin J Geophys (in Chinese) 45(2):255-262

Hou ZY, Guo CS, Wang JQ (2013) Surface sediments acoustic velocity and porosity correlation in Nansha sea area abyssal region. Mar Sci 37(7):77-82

Kanaun S, Levin V, Ronquillo G (2012) Detection of anisotropy parameters of cracked rocks in marine seismic studies. J Geophys Eng 9(9):39

Kennett BLN (1979) Theoretical reflection seismograms for elastic media. Geophys Prospect 27(2):301-321

Liu Y, Liu X (2015) Seafloor elastic parameters estimation based on AVO inversion. Mar Geophys Res 36(4):335-342

Liu Y, Tinivella U, Liu X (2015) An inversion method for seafloor elastic parameters. Geophysics 80(3):N11-N21

Long JJ, Li GZ, Zou DP (2014) Theoretical relations of longitudinal wave velocity and physical- mechanical properties for seafloor sediments and comparison. Acta Oceanol Sin (in Chinese) 36(7):111-117

Lu J, Yang Z, Wang Y (2015) Joint PP and PS AVA seismic inversion using exact Zoeppritz equations. Geophysics 80(5):R239-R250

Mallick S, Frazer LN (1991) Reflection/transmission coefficients and azimuthal anisotropy in marine seismic studies. Geophys J Int 105(1):241-252

Meng XM, Liu BH, Kan GM (2012) An experimental study on acoustic properties and their influencing factors of marine sediment in the southern Huanghai Sea. Acta Oceanol Sin (in Chinese) 34(6):74-83

Nguyen VH, Abdoulatuf A, Desceliers C (2016) A probabilistic study of reflection and transmission coefficients of random anisotropic elastic plates. Wave Motion 64:103-118

Ostrander WJ (1984) Plane-wave reflection coefficients for gas sands at non-normal angles of incidence. Geophysics 49(10): $1637-1648$

Rafipour B, Herrin E (1986) Phase offset indicator (POI): a study of phase shift versus offset and fluid content. Geophysics 51(3):679-688

Riedel M, Theilen F (2001) AVO investigations of shallow marine sediments. Geophys Prospect 49(2):198-212

Riedel M, Dosso SE, Beran L (2003) Uncertainty estimation for amplitude variation with offset (AVO) inversion. Geophysics 68(5):1485-1489

Ruan AG, Li JB, Chu FY et al (2006) AVO numerical simulation of gas hydrates reflectors beneath seafloor. Chin J Geophys. (in Chinese) 49(6):1826-1835

Ruud BO (2006) Ambiguous reflections coefficients for anelastic media. Stud Geophys Geod 30:479-498

Shuey RT (1985) A simplification of the Zoeppritz equations. Geophysics 50:609-614

Sidler R, Carcione JM (2007) Wave reflection at an anelastic transversely isotropic ocean bottom. Geophys 72(72):SM139SM146

Singh MC, Chakraborty N (2013) Reflection and refraction of P-, SVand thermal wave, at an initially stressed solid-liquid interface in generalized thermoelasticity. Appl Math Model 37(s1-2):463-475

Skopintseva L, Ayzenberg M, Landrø M (2011) Long-offset AVO inversion of $\mathrm{PP}$ reflections from plane interfaces using effective reflection coefficients. Geophysics 76(6):889-893

Sun PY, Lu XL, Li YP (2008) Elastic parameter AVO approximations and their applications. In: 78th Annual International Meeting, SEG, Expanded Abstracts, pp 523-527

Xu Y, Bancroft JC (1997) Joint AVO analysis of PP and PS seismic data. Crewes Research Report, pp 1-44

Yin XY, Zong ZY, Wu GC (2013) Improving seismic interpretation: a high-contrast approximation to the reflection coefficient of a plane longitudinal wave. Pet Sci 10(4):466-476

Zhao X, Shen HH (2013) Ocean wave transmission and reflection between two connecting viscoelastic ice covers: an approximate solution. Ocean Model 71(7):102-113

Zhu X, McMechan GA (2012) Elastic inversion of near- and postcritical reflections using phase variation with angle. Geophysics 77(4):R149-R159

Zoeppritz R (1919) On the reflection and propagation of seismic waves: erdbenwellen VIIIB. Göttinger Nachrichten I:66-84

Zong Z, Yin X, Wu G (2013) Multi-parameter nonlinear inversion with exact reflection coefficient equation. J Appl Geophys 98(11):21-32 